\title{
Partially Multiplicative Biquandles and Handlebody-Knots
}

\author{
Atsushi Ishii* Sam Nelson ${ }^{\dagger}$
}

\begin{abstract}
We introduce several algebraic structures related to handlebody-knots, including $G$-families of biquandles, partially multiplicative biquandles and group decomposable biquandles. These structures can be used to color the semiarcs in $Y$-oriented spatial trivalent graph diagrams representing $S^{1}$-oriented handlebody-knots to obtain computable invariants for handlebody-knots and handlebody-links. In the case of $G$-families of biquandles, we enhance the counting invariant using the group $G$ to obtain a polynomial invariant of handlebody-knots.
\end{abstract}

KEYwords: Handlebody-knots, biquandles, $G$-families of biquandles, partially multiplicative biquandles, group decomposable biquandles

2000 MSC: 57M27, 57M25

\section{Introduction}

Introduced in the early 1980s, quandles are algebraic structures which can be used to distinguish knots and links by counting colorings of arcs (the portions going from one under-crossing to another) in an oriented knot or link diagram by elements of a fixed quandle 2, 7, 10. In [3] and later 8, quandles were generalized to biquandles which can be used to distinguish oriented knots and links by counting colorings of the semiarcs (the portions going from one under-crossing or over-crossing to another) in an oriented knot or link diagram.

In previous work such as 5, 6, 9, quandles and related structures such as $G$-families of quandles and qualgebras were used to define invariants of spatial trivalent graphs and related structures such as handlebodyknots by coloring the arcs (now defined as portions going from one under-crossing or vertex to another). In this paper we generalize these structures to structures for coloring the semiarcs of handlebody-knot diagrams, now defined as the portions of the diagram between under-crossing points, over-crossing points, and vertices.

The paper is organized as follows. In Section 2 we review the basics of biquandles and the counting invariant. In Section 3 we introduce $n$-parallel biquandles. In Section 4 we extend the notion of $G$-families of quandles to the biquandle case. In Section 5 we introduce partially multiplicative biquandles and a special case, group decomposable biquandles. In Section 6 we discuss invariants defined using these structures and provide examples of their computation. We conclude in Section 7 with some questions for future research.

\section{Biquandles}

We begin with a definition. (See 1 for more).

Definition 2.1. A biquandle is a set $X$ with maps $\unrhd, \bar{\nabla}: X \times X \rightarrow X$ satisfying

(i) For all $x \in X, x \unrhd x=x \bar{\triangleright} x$,

\footnotetext{
*Email: aishii@math.tsukuba.ac.jp. Partially supported by JSPS KAKENHI Grant Number 15K04866

$\dagger$ Email: knots@esotericka.org. Partially Supported by Simons Foundation Collaboration Grant 316709
} 
(ii) For each $y \in X$, the maps $\alpha_{y}, \beta_{y}: X \rightarrow X$ and $S: X \times X \rightarrow X \times X$ defined by

$$
\alpha_{y}(x)=x \bar{\triangleright} y, \quad \beta_{y}(x)=x \unrhd y \quad \text { and } \quad S(x, y)=(y \bar{\triangleright} x, x \unrhd y)
$$

are bijective, and

(iii) The exchange laws are satisfied:

$$
\begin{aligned}
& (x \unrhd y) \unrhd(z \unrhd y) \quad=\quad(x \unrhd z) \unrhd(y \bar{\triangleright} z)
\end{aligned}
$$

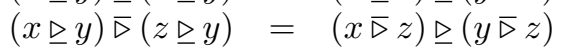

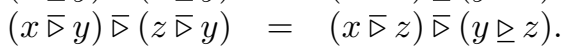

A biquandle in which $x \bar{\triangleright} y=x$ for all $x, y \in X$ is a quandle.

Example 1. For any set $X$ and bijection $\sigma: X \rightarrow X$, the operations $x \unrhd y=x \bar{\triangleright} y=\sigma(x)$ define a biquandle called a constant action biquandle.

Example 2. For any abelian group $A$ with automorphisms $s, t: A \rightarrow A$, the operations

$$
x \unrhd y=t(x-y)+s(y), \quad x \bar{\triangleright} y=s(x)
$$

define a biquandle called an Alexander biquandle.

Example 3. For any group $G$, the operations $x \unrhd y=y^{-1} x y$ and $x \bar{\triangleright} y=x$ define a biquandle (indeed, a quandle) structure known as the conjugation quandle of $G$.

The biquandle axioms are motivated by the Reidemeister moves for oriented knots and links. Specifically, if $X$ is a biquandle then an assignment of an element of $X$ to each semiarc in an oriented knot or link diagram is a biquandle coloring of the diagram if at every crossing we have
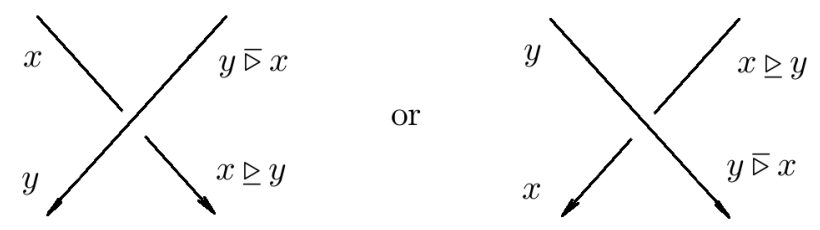

Recall that two knot or link diagrams represent ambient isotopic knots or links if and only if they differ by a sequence of Reidemeister moves:
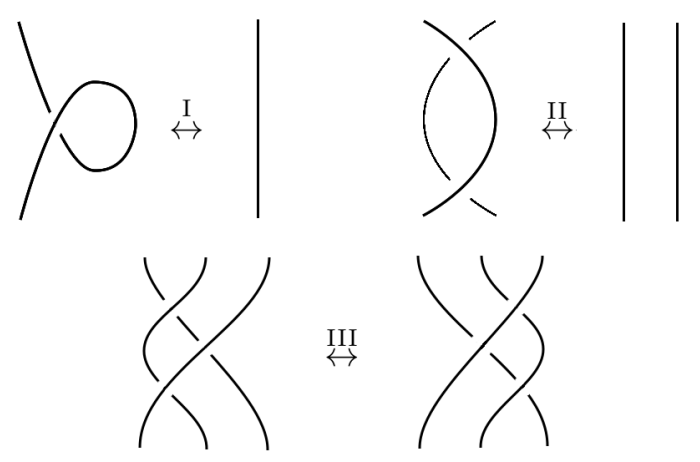

It is then easy to check the following standard result (see also [1]). 
Theorem 2.2. Let $D$ be an oriented knot or link diagram with a choice of biquandle coloring. Then for any Reidemeister move $\Omega$, there is a unique biquandle coloring of the diagram $D^{\prime}$ obtained from $D$ by applying $\Omega$ which agrees with the coloring on $D$ outside the neighborhood of the move.

Definition 2.3. Let $X$ be a biquandle and $D$ an oriented knot or link diagram. Then the set of biquandle colorings of $D$ by $X$ is denoted $\mathcal{C}_{X}(D)$.

Denote the cardinaility of $\mathcal{C}_{X}(D)$ by $\left|\mathcal{C}_{X}(D)\right|$. Then we have the following:

Corollary 2.4. Let $X$ be a finite biquandle. Then for any two diagrams $D, D^{\prime}$ of an oriented knot or link $L$, we have $\left|\mathcal{C}_{X}(D)\right|=\left|\mathcal{C}_{X}\left(D^{\prime}\right)\right|$.

Definition 2.5. For any biquandle $X$, the number $\left|\mathcal{C}_{X}(K)\right|$ of biquandle colorings of $K$ by $X$ is called the biquandle counting invariant of $K$ with respect to the biquandle $X$, denoted $\Phi_{X}^{\mathbb{Z}}(K)$.

Example 4. Let $X=\mathbb{Z}_{3}=\mathbb{Z} / 3 \mathbb{Z}$ and set $t=1$ and $s=2$; then $X$ is an Alexander biquandle with operations $x \unrhd y=x+y$ and $x \bar{\triangleright} y=2 x$. We can compute the biquandle counting invariant by row-reducing the matrices expressing the crossing relations over $\mathbb{Z}_{3}$. For example, the Hopf link

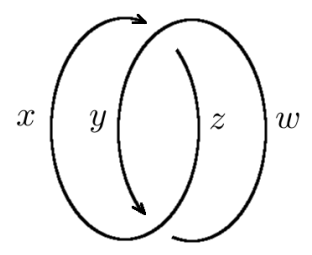

has crossing equations $x+y=z, 2 y=w, y+x=w, 2 x=z$ and thus $X$-coloring matrix which row-reduces over $\mathbb{Z}_{3}$ to

$$
\left[\begin{array}{llll}
1 & 1 & 2 & 0 \\
0 & 2 & 0 & 2 \\
1 & 1 & 0 & 2 \\
2 & 0 & 2 & 0
\end{array}\right] \longleftrightarrow\left[\begin{array}{llll}
1 & 0 & 0 & 1 \\
0 & 1 & 0 & 1 \\
0 & 0 & 1 & 2 \\
0 & 0 & 0 & 0
\end{array}\right]
$$

so $\mid \mathcal{C}_{X}$ (Hopf Link) $|=| X \mid=3$. The unlink of two circles has 9 colorings, and hence the invariant detects the non-triviality of the Hopf link.

\section{3 n-Parallel Biquandles}

We would like to extend biquandles to algebraic structures suitable for defining counting invariants for spatial trivalent graphs and their quotient structure, handlebody-knots. We will first develop some new notation.

Definition 3.1. Let $(X, \unrhd, \bar{\varnothing})$ be a biquandle. For $n>0$, we define

$$
\begin{array}{ll}
a \unrhd^{[0]} b=a, & a \triangleright^{[0]} b=a, \\
a \unrhd^{[n]} b=\left(a \unrhd^{[n-1]} b\right) \unrhd\left(b \unrhd^{[n-1]} b\right), \quad \text { and } & a \triangleright^{[n]} b=\left(a \triangleright^{[n-1]} b\right) \triangleright\left(b \triangleright^{[n-1]} b\right) .
\end{array}
$$

Example 5. Let $X$ be a biquandle. Then we have

$$
a \unrhd^{[1]} b=a \unrhd b, \quad a \unrhd^{[2]} b=(a \unrhd b) \unrhd(b \unrhd b), \quad a \unrhd^{[3]} b=((a \unrhd b) \unrhd(b \unrhd b)) \unrhd((b \unrhd b) \unrhd(b \unrhd b))
$$

et cetera.

Remark 3.2. If $(X, \unrhd, \bar{\nabla})$ is a quandle, i.e. a biquandle with $a \bar{\triangleright} b=a$ for all $a, b \in X$, then we have

$$
a \unrhd^{[n]} b=a \unrhd^{n} b=\beta_{b}^{n}(a) \quad \text { and } \quad a \triangleright^{[n]} b=a
$$

where $\beta_{y}(x)=x \unrhd y$. 
Let $(X, \unrhd, \bar{\nabla})$ be a biquandle. We will show via a series of lemmas that $\left(X, \unrhd^{[n]}, \bar{\triangleright}^{[n]}\right)$ is also a biquandle.

Lemma 3.3. For $m, n \geq 0$, we have

$$
\left(a \unrhd^{[m]} b\right) \unrhd^{[n]}\left(b \unrhd^{[m]} b\right)=a \unrhd^{[m+n]} b, \quad\left(a \triangleright^{[m]} b\right){\triangleright^{[n]}}^{[m}\left(b{\triangleright^{[m]}}^{[m}\right)=a \bar{\triangleright}^{[m+n]} b .
$$

Proof. The proof is by induction on $n$. When $n=0$, we have

$$
\left(a \unrhd^{[m]} b\right) \unrhd^{[0]}\left(b \unrhd^{[m]} b\right)=\left(a \unrhd^{[m]} b\right)=\left(a \unrhd^{[m+0]} b\right) .
$$

Now suppose that the equality holds for $n<k$. Then we have

$$
\begin{aligned}
\left(a \unrhd^{[m]} b\right) \unrhd^{[k]}\left(b \unrhd^{[m]} b\right) & =\left(\left(a \unrhd^{[m]} b\right) \unrhd^{[k-1]}\left(b \unrhd^{[m]} b\right)\right) \unrhd\left(\left(b \unrhd^{[m]} b\right) \unrhd^{[k-1]}\left(b \unrhd^{[m]} b\right)\right) \\
& =\left(a \unrhd^{[m+k-1]} b\right) \unrhd\left(b \unrhd^{[m+k-1]} b\right)=a \unrhd^{[m+k]} b
\end{aligned}
$$

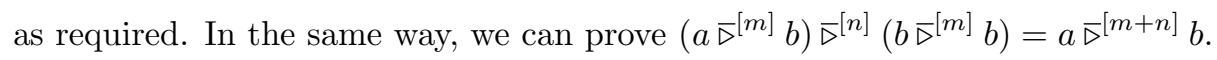

We now verify that $\left(X, \unrhd^{[n]}, \nabla^{[n]}\right)$ satisfies the biquandle axioms.

Lemma 3.4. For $n \geq 0$, we have $a \unrhd^{[n]} a=a \unrhd^{[n]} a$.

Proof. Again, we proceed by induction on $n$. When $n=0$, we have $a \unrhd^{[0]} a=a=a{\triangleright^{[0]}}^{[0}$. Then suppose that the equality holds for $n<k$. Then we have

$$
a \unrhd^{[k]} a=\left(a \unrhd^{[k-1]} a\right) \unrhd\left(a \unrhd^{[k-1]} a\right)=\left(a \bar{\triangleright}^{[k-1]} a\right) \bar{\triangleright}\left(a \bar{\triangleright}^{[k-1]} a\right)=a{\triangleright^{[k]}}^{[k}
$$

as required.

Next, we verify the exchange laws.

Lemma 3.5. For $m, n \geq 0$, we have

$$
\begin{aligned}
& \left(a \unrhd^{[m]} b\right) \unrhd^{[n]}\left(c \bar{\triangleright}^{[m]} b\right)=\left(a \unrhd^{[n]} c\right) \unrhd^{[m]}\left(b \unrhd^{[n]} c\right),
\end{aligned}
$$

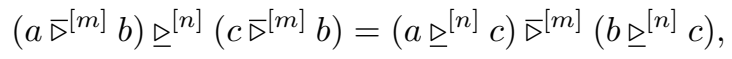

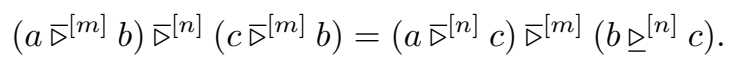

Proof. When $n=0$, we have

$$
\begin{aligned}
& \left(a \unrhd^{[m]} b\right) \unrhd^{[0]}\left(c \Sigma^{\bar{D}^{[m]}} b\right)=a \unrhd^{[m]} b=\left(a \unrhd^{[0]} c\right) \unrhd^{[m]}\left(b \unrhd^{[0]} c\right), \\
& \left(a \Sigma^{[m]} b\right) \unrhd^{[0]}\left(c \triangleright^{[m]} b\right)=a \Sigma^{[m]} b=\left(a \unrhd^{[0]} c\right) \triangleright^{[m]}\left(b \unrhd^{[0]} c\right) \text {, }
\end{aligned}
$$

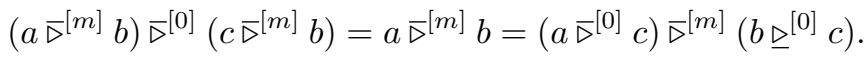

When $m=0$, we have

$$
\begin{aligned}
& \left(a \unrhd^{[0]} b\right) \unrhd^{[n]}\left(c{\triangleright^{[0]}}^{[0)}=a \unrhd^{[n]} c=\left(a \unrhd^{[n]} c\right) \unrhd^{[0]}\left(b \unrhd^{[n]} c\right),\right.
\end{aligned}
$$

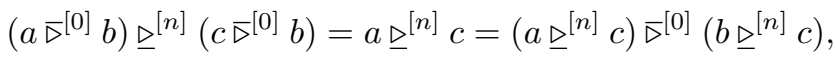

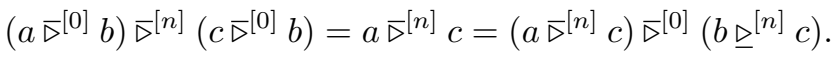

Suppose that the three equalities hold for $n=1, m<k$. Then we have

$$
\begin{aligned}
\left(a \unrhd^{[k]} b\right) \unrhd\left(c \triangleright^{[k]} b\right) & =\left(\left(a \unrhd^{[k-1]} b\right) \unrhd\left(b \unrhd^{[k-1]} b\right)\right) \unrhd\left(\left(c \triangleright^{[k-1]} b\right) \triangleright\left(b \triangleright^{[k-1]} b\right)\right) \\
& =\left(\left(a \unrhd^{[k-1]} b\right) \unrhd\left(c \bar{\triangleright}^{[k-1]} b\right)\right) \unrhd\left(\left(b \unrhd^{[k-1]} b\right) \unrhd\left(c \triangleright^{[k-1]} b\right)\right) \\
& =\left((a \unrhd c) \unrhd^{[k-1]}(b \unrhd c)\right) \unrhd\left((b \unrhd c) \unrhd^{[k-1]}(b \unrhd c)\right) \\
& =(a \unrhd c) \unrhd^{[k]}(b \unrhd c) .
\end{aligned}
$$


In the same way, we have

$$
\begin{aligned}
& \left(a \bar{\triangleright}^{[m]} b\right) \unrhd\left(c \bar{\triangleright}^{[m]} b\right)=(a \unrhd c) \bar{\triangleright}^{[m]}(b \unrhd c), \\
& \left(a \bar{\triangleright}^{[m]} b\right) \bar{\triangleright}\left(c \bar{\triangleright}^{[m]} b\right)=(a \bar{\triangleright} c) \bar{\triangleright}^{[m]}(b \unrhd c) .
\end{aligned}
$$

Suppose that the three equalities hold for $n<k$. Then we have

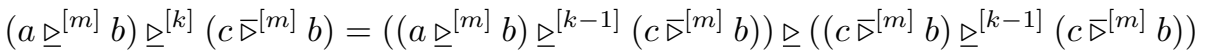

$$
\begin{aligned}
& =\left(\left(a \unrhd^{[k-1]} c\right) \unrhd^{[m]}\left(b \unrhd^{[k-1]} c\right)\right) \unrhd\left(\left(c \unrhd^{[k-1]} c\right) \bar{\triangleright}^{[m]}\left(b \unrhd^{[k-1]} c\right)\right) \\
& =\left(\left(a \unrhd^{[k-1]} c\right) \unrhd\left(c \unrhd^{[k-1]} c\right)\right) \unrhd^{[m]}\left(\left(b \unrhd^{[k-1]} c\right) \unrhd\left(c \unrhd^{[k-1]} c\right)\right) \\
& =\left(a \unrhd^{[k]} c\right) \unrhd^{[m]}\left(b \unrhd^{[k]} c\right) \text {. }
\end{aligned}
$$

In the same way, we have

$$
\begin{aligned}
& \left(a \triangleright^{[m]} b\right) \unrhd^{[n]}\left(c \triangleright^{[m]} b\right)=\left(a \unrhd^{[n]} c\right) \triangleright^{[m]}\left(b \unrhd^{[n]} c\right) \quad \text { and } \\
& \left(a \triangleright^{[m]} b\right) \triangleright^{[n]}\left(c{\triangleright^{[m]}}^{[m}\right)=\left(a \triangleright^{[n]} c\right) \triangleright^{[m]}\left(b \unrhd^{[n]} c\right)
\end{aligned}
$$

as required.

Lemma 3.6. Let $m, n \geq 0$. Then

(i) The maps $\unrhd^{[n]} a: X \rightarrow X,{\triangleright^{[n]}}^{[n}: X \rightarrow X$ are bijections for each $a \in X$.

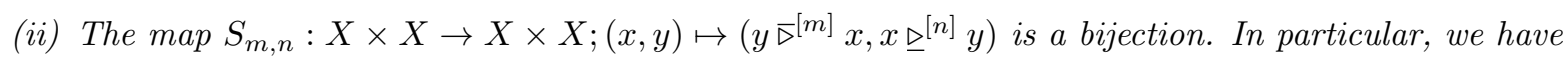

$$
a \unrhd^{[n]} a=b \unrhd^{[n]} b \Leftrightarrow a=b \Leftrightarrow a \triangleright^{[n]} a=b{\triangleright^{[n]}}^{[n}
$$

Proof. (i) From $x \unrhd^{[n-1]} a=\left(x \unrhd^{[n]} a\right) \unrhd^{-1}\left(a \unrhd^{[n-1]} a\right)$, the map $\unrhd^{[n]} a: X \rightarrow X$ is bijective for each $a \in X$. In the same way, we see that the map $\bar{\triangleright}^{[n]} a: X \rightarrow X$ is bijective for each $a \in X$.

(ii) Set $a_{i, j}=\left(x \unrhd^{[i]} y\right) \unrhd^{[j]}\left(x \unrhd^{[i]} y\right)$ and $a^{i, j}=\left(y{\triangleright^{[j]}}^{[j) \triangleright^{[i]}}\left(y{\triangleright^{[j]}}^{[j}\right)\right.$. Then

$$
S_{m, n}\left(a_{0,0}, a^{0,0}\right)=\left(a^{0, m}, a_{n, 0}\right) .
$$

Since $a_{i, j}=a_{i, 0} \unrhd^{[j]} a_{i, 0}, a_{n, 1}, \ldots, a_{n, m-1}$ are uniquely determined from $a_{n, 0}$, and since $a^{i, j}=a^{0, j}{\triangleright^{[i]}}^{0, j}$, $a^{1, m}, \ldots, a^{n-1, m}$ are uniquely determined from $a^{0, m}$. Then

$$
\begin{aligned}
& a_{i, j} \unrhd a^{i, j}=\left(\left(x \unrhd^{[i]} y\right) \unrhd^{[j]}\left(x \unrhd^{[i]} y\right)\right) \unrhd^{[1]}\left(\left(y{\Sigma^{[j]}}^{[j)} \unrhd^{[i]}\left(y \Sigma^{[j]} x\right)\right)\right. \\
& =\left(\left(x \unrhd^{[i]} y\right) \unrhd^{[j]}\left(x \unrhd^{[i]} y\right)\right) \unrhd^{[1]}\left(\left(y \unrhd^{[i]} y\right) \triangleright^{[j]}\left(x \unrhd^{[i]} y\right)\right) \\
& =\left(\left(x \unrhd^{[i]} y\right) \unrhd^{[1]}\left(y \unrhd^{[i]} y\right)\right) \unrhd^{[j]}\left(\left(x \unrhd^{[i]} y\right) \unrhd^{[1]}\left(y \unrhd^{[i]} y\right)\right) \\
& =\left(x \unrhd^{[i+1]} y\right) \unrhd^{[j]}\left(x \unrhd^{[i+1]} y\right)=a_{i+1, j},
\end{aligned}
$$

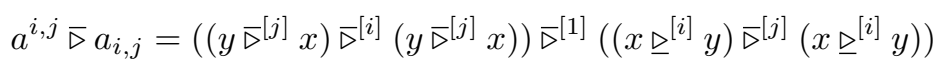

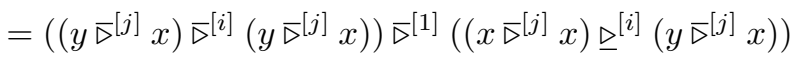

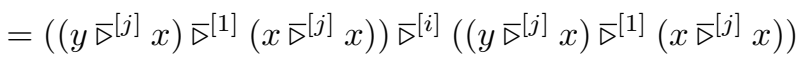

$$
\begin{aligned}
& =\left(y{\triangleright^{[j+1]}}^{[j) \triangleright^{[i]}}\left(y \triangleright^{[j+1]} x\right)=a^{i, j+1}\right. \text {. }
\end{aligned}
$$

Since $S\left(a_{i, j}, a^{i, j}\right)=\left(a^{i, j} \bar{\triangleright} a_{i, j}, a_{i, j} \unrhd a^{i, j}\right)=\left(a^{i, j+1}, a_{i+1, j}\right)$, we have $S^{-1}\left(a^{i, j+1}, a_{i+1, j}\right)=\left(a_{i, j}, a^{i, j}\right)$. Moreover, $a_{n-1,0}$ is uniquely determined from $a_{n, 0}, \ldots, a_{n, m-1}$, since we have

$$
\begin{aligned}
S^{-1}\left(a^{n-1, m}, a_{n, m-1}\right) & =\left(a_{n-1, m-1}, a^{n-1, m-1}\right), \\
S^{-1}\left(a^{n-1, m-1}, a_{n, m-2}\right) & =\left(a_{n-1, m-2}, a^{n-1, m-2}\right), \\
S^{-1}\left(a^{n-1, m-2}, a_{n, m-3}\right) & =\left(a_{n-1, m-3}, a^{n-1, m-3}\right), \ldots \\
S^{-1}\left(a^{n-1,1}, a_{n, 0}\right) & =\left(a_{n-1,0}, a^{n-1,0}\right)
\end{aligned}
$$


and $a^{0, m-1}$ is uniquely determined from $a^{0, m}, \ldots, a^{n-1, m}$, since we have

$$
\begin{aligned}
S^{-1}\left(a^{n-1, m}, a_{n, m-1}\right) & =\left(a_{n-1, m-1}, a^{n-1, m-1}\right), \\
S^{-1}\left(a^{n-2, m}, a_{n-1, m-1}\right) & =\left(a_{n-2, m-1}, a^{n-2, m-1}\right), \\
S^{-1}\left(a^{n-3, m}, a_{n-2, m-1}\right) & =\left(a_{n-3, m-1}, a^{n-3, m-1}\right), \ldots \\
S^{-1}\left(a^{0, m}, a_{1, m-1}\right) & =\left(a_{0, m-1}, a^{0, m-1}\right) .
\end{aligned}
$$

Repeating this, we see that $a_{0,0}, a^{0,0}$ are uniquely determined from $a_{n, 0}, a^{0, m}$.

Definition 3.7. Fix $n \geq 0$. We call $\left(X, \unrhd^{[n]}, \triangleright^{[n]}\right)$ the $n$-parallel biquandle of $(X, \unrhd, \bar{\nabla})$.

Example 6. Consider the constant action biquandle $X=\mathbb{Z}_{m}$ with $x \bar{\triangleright} y=x \unrhd y=x+1$. Then the $n$-parallel biquandle of $X$ is $\mathbb{Z}_{m}$ with $x \bar{\triangleright} y=x \unrhd y=x+n$.

Example 7. More generally, if $\sigma: X \rightarrow X$ is a bijection then the $n$-parallel biquandle of the constant action biquandle $X$ with $x \unrhd y=x \bar{\triangleright} y=\sigma(x)$ is $X$ with $x \unrhd^{[n]} y=x \bar{\triangleright}^{[n]} y=\sigma^{n}(x)$.

Proposition 3.8. Let $X$ be an Alexander biquandle with operations

$$
x \unrhd y=t x+(s-t) y \quad \text { and } \quad x \bar{\triangleright} y=s x .
$$

Then the $n$-parallel biquandle of $X$ is the set $X$ with biquandle operations

$$
x \unrhd^{[n]} y=t^{n} x+\left(s^{n}-t^{n}\right) y \quad \text { and } \quad x \Sigma^{[n]} y=s^{n} x .
$$

Proof. As a base case, if $n=1$ we have

$$
x \unrhd^{[1]} y=x \unrhd y=t^{1} x+\left(s^{1}-t^{1}\right) y \quad \text { and } \quad x \bar{\triangleright}^{[1]} y=x \bar{\triangleright} y=s^{1} x .
$$

Now, suppose $x \unrhd^{[n-1]} y=t^{n-1} x+\left(s^{n-1}-t^{n-1} y\right)$; then we have

$$
\begin{aligned}
x \unrhd^{[n]} y & =\left(x \unrhd^{[n-1]} y\right) \unrhd\left(y \unrhd^{[n-1]} y\right) \\
& =t\left(t^{n-1} x+\left(s^{n-1}-t^{n-1}\right) y\right)+(s-t)\left(t^{n-1} y+\left(s^{n-1}-t^{n-1}\right) y\right) \\
& =t^{n} x+\left[t\left(s^{n-1}-t^{n-1}\right)+(s-t) t^{n-1}+(s-t)\left(s^{n-1}-t^{n-1}\right)\right] y \\
& =t^{n} x+\left[t s^{n-1}-t^{n}+s t^{n-1}-t^{n}+s^{n}-s t^{n-1}-t s^{n-1}+t^{n}\right] y \\
& =t^{n} x+\left(s^{n}-t^{n}\right) y
\end{aligned}
$$

while $x{\triangleright^{[n]}}^{n}=s^{n} x$ as required.

\section{$4 \quad$ G-Families of Biquandles}

In this section we generalize a definition from 6 to the case of biquandles.

Definition 4.1. Let $G$ be a group and $X$ a set. We say that $\left(X,\left(\unrhd^{g}\right)_{g \in G},\left(\bar{\triangleright}^{g}\right)_{g \in G}\right)$ is a $G$-family of biquandles if

(i) $a \unrhd^{g} a=a \bar{\triangleright}^{g} a(\forall g \in G, \forall a \in X)$

(ii) $\unrhd^{g} a: X \rightarrow X ; x \mapsto x \unrhd^{g} a$ is a bijection $(\forall g \in G, \forall a \in X)$

$\bar{\triangleright}^{g} a: X \rightarrow X ; x \mapsto x \bar{\triangleright}^{g} a$ is a bijection $(\forall g \in G, \forall a \in X)$

$S_{g, h}: X \times X \rightarrow X \times X ;(x, y) \mapsto\left(y \bar{\triangleright}^{g} x, x \unrhd^{h} y\right)$ is bijective $(\forall g, h \in G)$ 
(iii) $\left(a \unrhd^{g} b\right) \unrhd^{h}\left(c \bar{\triangleright}^{g} b\right)=\left(a \unrhd^{h} c\right) \unrhd^{h^{-1} g h}\left(b \unrhd^{h} c\right)$

$\left(a \bar{\triangleright}^{g} b\right) \unrhd^{h}\left(c \bar{\triangleright}^{g} b\right)=\left(a \unrhd^{h} c\right) \bar{\triangleright}^{h^{-1} g h}\left(b \unrhd^{h} c\right)$

$\left(a \bar{\triangleright}^{g} b\right) \bar{\triangleright}^{h}\left(c \bar{\triangleright}^{g} b\right)=\left(a \bar{\triangleright}^{h} c\right) \bar{\triangleright}^{h^{-1} g h}\left(b \unrhd^{h} c\right)(\forall g, h \in G, \forall a, b, c \in X) \quad$ and

(iv) $a \unrhd^{g h} b=\left(a \unrhd^{g} b\right) \unrhd^{h}\left(b \unrhd^{g} b\right)$

$a \bar{\triangleright}^{g h} b=\left(a \bar{\triangleright}^{g} b\right) \bar{\triangleright}^{h}\left(b \bar{\triangleright}^{g} b\right)(\forall g, h \in G, \forall a, b \in X)$

Definition 4.2. Let $X$ be a biquandle. We define the idempotency index and type of $X$ by

$\operatorname{idem} X=\min \left\{n>0 \mid a \unrhd^{[n]} a=a(\forall a \in X)\right\}$ and

type $X=\min \left\{n>0 \mid a \unrhd^{[n]} b=a=a \Sigma^{[n]} b(\forall a, b \in X)\right\}$.

Lemma 4.3. Let $m \geq n \geq 0$. Let $X$ be a biquandle such that $\operatorname{idem} X$, type $X<\infty$. Then we have

(i) idem $X \mid(m-n) \Rightarrow a \unrhd^{[m]} a=a \unrhd^{[n]} a$ and $a \bar{\triangleright}^{[m]} a=a \bar{\triangleright}^{[n]} a(\forall a \in X)$,

(ii) type $X \mid(m-n) \Rightarrow a \unrhd^{[m]} b=a \unrhd^{[n]} b$ and $a \bar{\triangleright}^{[m]} b=a \triangleright^{[n]} b(\forall a, b \in X)$ and

(iii) idem $X \mid$ type $X$.

Proof. (i) First, we compute

$$
\begin{aligned}
a \unrhd^{[m]} a=a \unrhd^{[m]} a & =a \unrhd^{[n+k \operatorname{idem} X]} a \\
& =\left(a \unrhd^{[\operatorname{idem} X]} a\right) \unrhd^{[n+(k-1) \operatorname{idem} X]}\left(a \unrhd^{[\operatorname{idem} X]} a\right) \\
& =a \unrhd^{[n+(k-1) \operatorname{idem} X]} a=\cdots=a \unrhd^{[n]} a=a \triangleright^{[n]} a .
\end{aligned}
$$

(ii) Next, we have

$$
\begin{aligned}
a \unrhd^{[m]} b & =a \unrhd^{[n+k \text { type } X]} b \\
& =\left(a \unrhd^{[\text {type } X]} b\right) \unrhd^{[n+(k-1) \operatorname{type} X]}\left(b \unrhd^{[\text {type } X]} b\right) \\
& =a \unrhd^{[n+(k-1) \text { type } X]} b=\cdots=a \unrhd^{[n]} b .
\end{aligned}
$$

In the same way, we have $a \Sigma^{[m]} b=a \Sigma^{[n]} b$.

(iii) From (i), we have

$$
\left\{n \in \mathbb{Z}_{\geq 0} \mid a \unrhd^{[n]} a=a(\forall a \in X)\right\}=(\operatorname{idem} X) \mathbb{Z}_{\geq 0} .
$$

It follows from $a \unrhd^{[\operatorname{type} X]} a=a$, that type $X \in(\operatorname{idem} X) \mathbb{Z}_{\geq 0}$.

Remark 4.4. For $n \in \mathbb{Z}_{\text {type } X}$ and $a \unrhd^{[n]} b, a \triangleright^{[n]} b$ are well-defined.

Theorem 4.5. Let $(X, \underline{\unrhd}, \bar{\nabla})$ be a biquandle with type $X<\infty$. Set $G=\mathbb{Z}_{\text {type } X}$. Then $\left(X,\left(\underline{(}^{[n]}\right)_{n \in G},\left(\bar{\triangleright}^{[n]}\right)_{n \in G}\right)$ is a $G$-family of biquandles called the $G$-family associated to $X$.

Proof. This follows from Lemmas $3.3,3.4,3.5$ and 3.6.

Proposition 4.6. Let $X$ be a finite biquandle. Then

$$
\operatorname{idem} X<\infty \text { and type } X<\infty \text {. }
$$


Proof. First, set $n_{a}=\min \left\{n>0 \mid a \unrhd^{[n]} a=a\right\}$. Then since $\left|\left\{a \unrhd^{[n]} a \mid n \in \mathbb{Z}_{\geq 0}\right\}\right|<\infty, \exists m, n>0(m>n)$ such that $a \unrhd^{[m]} a=a \unrhd^{[n]} a$. Since

$$
\left(a \unrhd^{[m-n]} a\right) \unrhd^{[n]}\left(a \unrhd^{[m-n]} a\right)=a \unrhd^{[m]} a=a \unrhd^{[n]} a,
$$

we have $a \unrhd^{[m-n]} a=a$ and therefore $n_{a} \leq m-n<\infty$. Since

$$
a \unrhd^{\left[k n_{a}\right]} a=\left(a \unrhd^{\left[n_{a}\right]} a\right) \unrhd^{\left[(k-1) n_{a}\right]}\left(a \unrhd^{\left[n_{a}\right]} a\right)=a \unrhd^{\left[(k-1) n_{a}\right]} a=\cdots=a,
$$

we have idem $X \leq \operatorname{lcm}\left(\left\{n_{a} \mid a \in X\right\}\right)<\infty$ as required.

Next, set $\underline{n}_{b}=\min \left\{n>0 \mid\left(\underline{\unrhd}^{\left[n_{b}\right]} b\right)^{n}=\operatorname{id}_{X}\right\}$ and $\bar{n}_{b}=\min \left\{n>0 \mid\left(\bar{\triangleright}^{\left[n_{b}\right]} b\right)^{n}=\operatorname{id}_{X}\right\}$. Since $X$ is finite, we have $\underline{n}_{b}, \bar{n}_{b}<\infty$. Then

$$
\begin{aligned}
& a \unrhd^{\left[k \underline{n}_{b} n_{b}\right]} b=\left(a \unrhd^{\left[n_{b}\right]} b\right) \unrhd^{\left[\left(k \underline{n}_{b}-1\right) n_{b}\right]}\left(b \unrhd^{\left[n_{b}\right]} b\right) \\
& =\left(a \unrhd^{\left[n_{b}\right]} b\right) \unrhd^{\left[\left(k \underline{n}_{b}-1\right) n_{b}\right]} b=\cdots=a \underbrace{\left[\unrhd^{\left[n_{b}\right]} b \cdots \unrhd^{\left[n_{b}\right]} b\right.}_{k \underline{n}_{b}}=a .
\end{aligned}
$$

In the same way, we see $a \bar{\triangleright}^{\left[k \bar{n}_{b} n_{b}\right]} b=a$. Therefore type $X \leq \operatorname{lcm}\left(\left\{\underline{n}_{b} n_{b}, \bar{n}_{b} n_{b} \mid b \in X\right\}\right)<\infty$ as required.

Example 8. Let $X=\mathbb{Z}_{5}$ and set $t=2$ and $s=3$. Then we have Alexander biquandle operations

$$
\begin{array}{lll}
x \unrhd^{[1]} y=2 x+(3-2) y=2 x+y, & x \bar{\triangleright}^{[1]} y=3 x \\
x \unrhd^{[2]} y=4 x+(9-4) y=4 x, & x \Sigma^{[2]} y=9 x=4 x \\
x \unrhd^{[3]} y=3 x+(27-8) y=3 x+4 y, & x \Sigma^{[3]} y=12 x=2 x \\
x \unrhd^{[4]} y=x+(81-16) y=x, & x \bar{\triangleright}^{[4]} y=6 x=x
\end{array}
$$

so $(X, \unrhd, \bar{\triangleright})$ has type 4 ; thus we have a $\mathbb{Z}_{4}$-family of biquandles associated to $X$.

Example 9. Let $X=\mathbb{Z}_{2}$ with operations $x \unrhd y=x \bar{\triangleright} y=x+1$. Then we have

$$
\begin{array}{ll}
x \unrhd^{[1]} y=x+1 & x{\triangleright^{[1]}}^{[1}=x+1 \\
x \unrhd^{[2]} y=(x+1)+1=x, & x \bar{\triangleright}^{[2]} y=(x+1)+1=x
\end{array}
$$

so $(X, \unrhd, \bar{\triangleright})$ has type 2 ; thus we have a $\mathbb{Z}_{2}$-family of biquandles associated to $X$.

\section{Partially Multiplicative Biquandles}

In this section we will extend the idea of biquandle colorings to $S^{1}$-oriented handlebody-knots represented by $Y$-oriented spatial trivalent graph diagrams by adding a new operation at vertices. Recall that the 
Reidemeister moves for handlebody-knots are given by

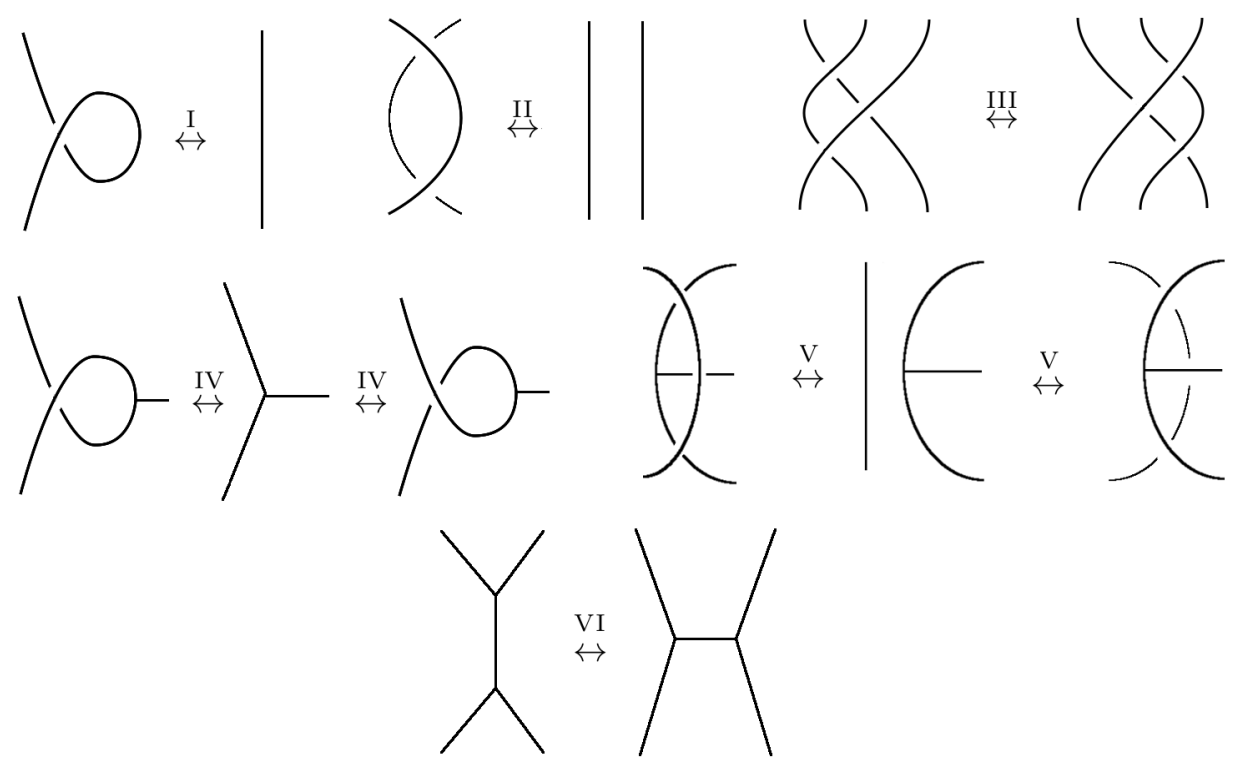

and that spatial trivalent graph diagrams represent ambient isotopic spatial trivalent graphs if they are related by moves I, II, III, IV and V, while including the diagrammatic move VI (corresponding to the spatial IH move) yields handlebody-knots. See [4,6] for more. A Y-orientation of a spatial trivalent graph diagram is a choice of direction for each edge in the underlying spatial graph such that no vertex is a source or a sink. An $S^{1}$-orientation of a handlebody-knot corresponds to a $Y$-orientation of a representative spatial trivalent graph; see [4] for more.

Definition 5.1. Let $(X, \unrhd, \bar{\nabla})$ be a biquandle, $D \subset X \times X$ a subset of the Cartesian product of $X$ with itself, and - a map from $D$ to $X$ called a partial multiplication. Then we say that $(X, \unrhd, \bar{\nabla}, \cdot: D \rightarrow X ;(a, b) \mapsto a b)$ is a partially multiplicative biquandle if

(i) $x \mapsto a x, x \mapsto x b$ are injective,

(ii) $(a, b \unrhd a) \in D \Leftrightarrow(b, a \bar{\triangleright} b) \in D \Rightarrow a(b \unrhd a)=b(a \triangleright b)$,

(iii) $(a, b) \in D \Leftrightarrow(a \unrhd x, b \unrhd(x \triangleright a)) \in D \Leftrightarrow(a \triangleright x, b \triangleright(x \unrhd a)) \in D \Rightarrow$

$$
\begin{aligned}
& x \unrhd(a b)=(x \unrhd a) \unrhd b, \quad(a b) \unrhd x=(a \unrhd x)(b \unrhd(x \bar{D} a)),
\end{aligned}
$$

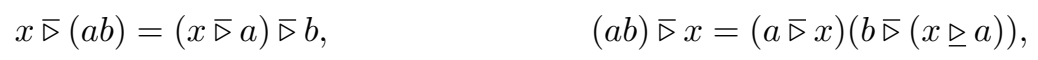

(iv) $(a, b),(a b, c) \in D \Leftrightarrow(b, c),(a, b c) \in D \Rightarrow(a b) c=a(b c)$ and

(v) $(a, b),(c, d) \in D, a b=c d \Leftrightarrow \exists e \in X$ such that $(a, e),(e, d) \in D, a e=c, e d=b$

If $D=\bigcup_{\lambda \in \Lambda} G_{\lambda} \times G_{\lambda}$ for a family of groups $\left\{G_{\lambda} \mid \lambda \in \Lambda\right\}$ with group operation $(a, b) \mapsto a b$, then we say $(X, \unrhd, \bar{\nabla}, \cdot)$ is a group decomposable biquandle.

Partially multiplicative biquandles can be used to extend biquandle colorings to handlebody-knots represented by spatial trivalent graphs with $Y$-orientations, i.e. directed trivalent graphs in $\mathbb{R}^{3}$ without sources or sinks. Given such a diagram $\Gamma$ and a partially multiplicative biquandle $X$, an assignment of elements of 
$X$ to the semiarcs of $\Gamma$ is an $X$-coloring if at each crossing and vertex we have the following:
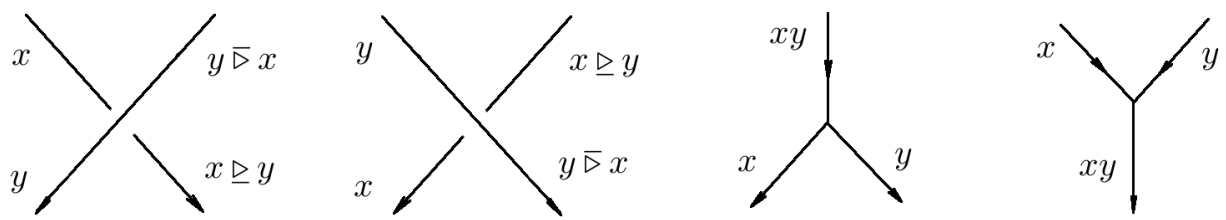

We then have:

Proposition 5.2. Let $\Gamma$ be a $Y$-oriented spatial trivalent graph diagram with an $X$-coloring by a partially multiplicative biquandle $X$. Then for any diagram $\Gamma^{\prime}$ obtained from $\Gamma$ by a handlebody-knot Reidemeister move, there is a unique $X$-coloring of $\Gamma^{\prime}$ agreeing with the coloring on $\Gamma$ outside the neighborhood of the move.

Proof. This is a matter of checking the Reidemeister moves for $Y$-oriented spatial trivalent graphs representing handlebody-knots and comparing the axioms in definition 5.1. Invariance under moves I, II and III is well-known; see [1], for instance. For each of the remaining moves, we illustrate with a choice of Y-orientation; the other cases are similar.
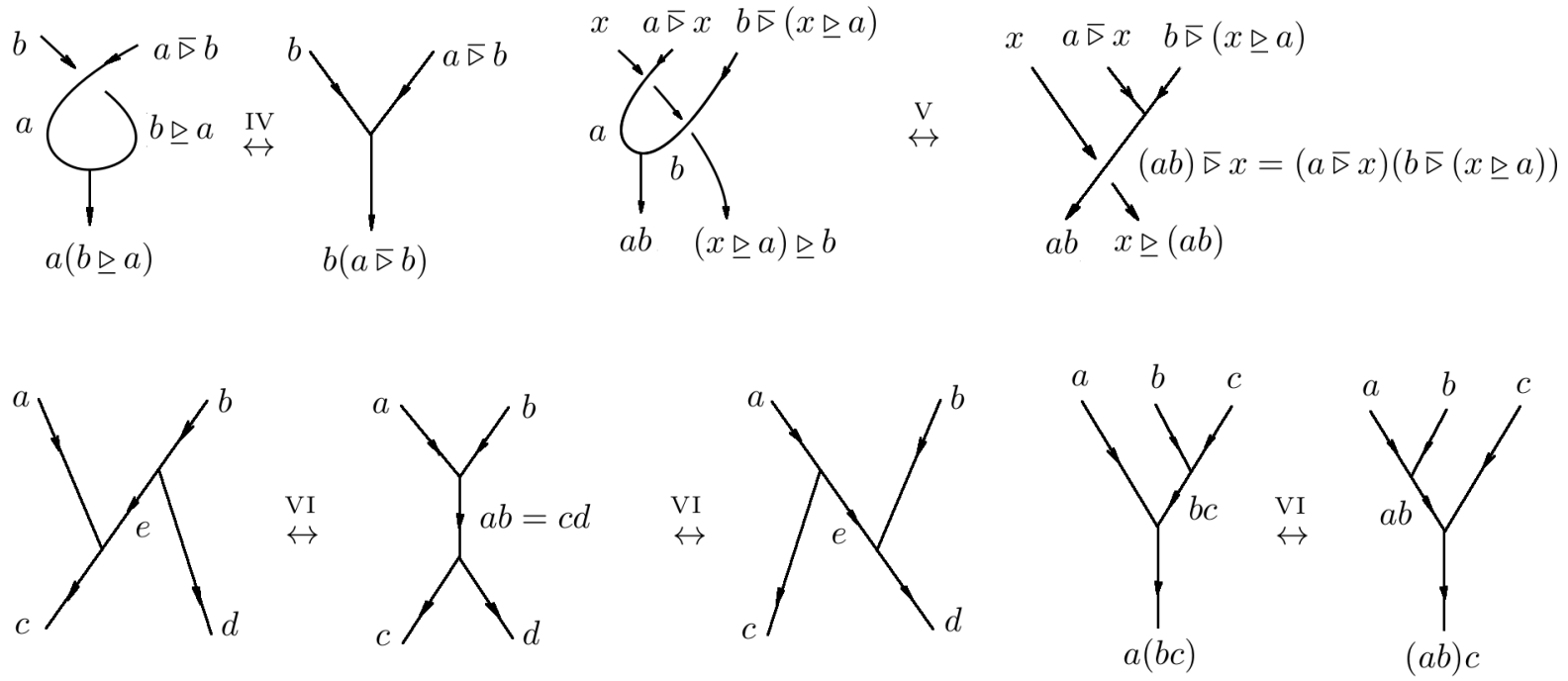

Corollary 5.3. The number of $X$-colorings of a $Y$-oriented spatial trivalent graph diagram representing an $S^{1}$-oriented handlebody-knot by a partially multiplicative biquandle $X$ is invariant under the handlebody-knot Reidemeister moves.

We've already seen our main example of partially multiplicative biquandles: $G$-families of biquandles.

Proposition 5.4. Let $\left(X, \unrhd^{g}, \bar{\triangleright}^{g}\right)$ be a G-family of biquandles. Set

$$
Q=X \times G \quad \text { and } \quad D=\left\{\left((a, g),\left(a \unrhd^{g} a, h\right)\right) \mid a \in X, g, h \in G\right\}
$$

and define

$$
\begin{aligned}
(a, g) \unrhd(b, h) & =\left(a \unrhd^{h} b, h^{-1} g h\right), \\
(a, g) \triangleright(b, h) & =\left(a \triangleright^{h} b, g\right) \text { and } \\
(a, g) \cdot\left(a \unrhd^{g} a, h\right) & =(a, g h) .
\end{aligned}
$$

Then, $(Q, \unrhd, \bar{\triangleright}, \cdot)$ is a partially multiplicative biquandle. 
Proof. This is a matter of verifying that the axioms of a partially multiplicative biquandle are satisfied. We must first show that $(Q, \unrhd, \bar{\triangleright})$ is a biquandle. We have

$$
(a, g) \unrhd(a, g)=\left(a \unrhd^{g} a, g^{-1} g g\right)=\left(a \bar{\triangleright}^{g} a, g\right)=(a, g) \triangleright(a, g)
$$

so the first biquandle axiom is satisfied. Since $\unrhd^{g} a, \bar{\triangleright}^{g} a: X \rightarrow X$ is bijective, $\unrhd(a, g): X \times G \rightarrow X \times G ;(x, k) \mapsto$ $\left(x \unrhd^{g} a, g^{-1} k g\right)$ and $\bar{\triangleright}(a, g): X \times G \rightarrow X \times G ;(x, k) \mapsto\left(x \bar{\triangleright}^{g} a, k\right)$ are bijective. Since $S_{g, h}: X \times X \rightarrow$ $X \times X ;(x, y) \mapsto\left(y \bar{\triangleright}^{g} x, x \unrhd^{h} y\right)$ is bijective, $S: Q \times Q \rightarrow Q \times Q ;((x, g),(y, h)) \mapsto\left(\left(y \triangleright^{g} x, h\right),\left(x \unrhd^{h} y, h^{-1} g h\right)\right)$ is bijective, and the second biquandle axiom is satisfied. Verifying the exchange laws, we have

$$
\begin{aligned}
& ((a, g) \unrhd(b, h)) \unrhd((c, k) \triangleright(b, h))=\left(a \unrhd^{h} b, h^{-1} g h\right) \unrhd\left(c \nabla^{h} b, k\right) \\
& =\left(\left(a \unrhd^{h} b\right) \unrhd^{k}\left(c \bar{\triangleright}^{h} b\right), k^{-1} h^{-1} g h k\right) \\
& =\left(\left(a \unrhd^{k} c\right) \unrhd^{k^{-1} h k}\left(b \unrhd^{k} c\right), k^{-1} h^{-1} g h k\right) \\
& =\left(a \unrhd^{k} c, k^{-1} g k\right) \unrhd\left(b \unrhd^{k} c, k^{-1} h k\right) \\
& =((a, g) \unrhd(c, k)) \unrhd((b, h) \unrhd(c, k)), \\
& ((a, g) \bar{\nabla}(b, h)) \unrhd((c, k) \bar{\triangleright}(b, h))=\left(a \bar{\triangleright}^{h} b, g\right) \unrhd\left(c \bar{\triangleright}^{h} b, k\right) \\
& =\left(\left(a \bar{\triangleright}^{h} b\right) \unrhd^{k}\left(c \bar{\triangleright}^{h} b\right), k^{-1} g k\right) \\
& =\left(\left(a \unrhd^{k} c\right) \triangleright^{k^{-1} h k}\left(b \unrhd^{k} c\right), k^{-1} g k\right) \\
& =\left(a \unrhd^{k} c, k^{-1} g k\right) \triangleright\left(b \unrhd^{k} c, k^{-1} h k\right) \\
& =((a, g) \unrhd(c, k)) \bar{\triangleright}((b, h) \unrhd(c, k))
\end{aligned}
$$

and

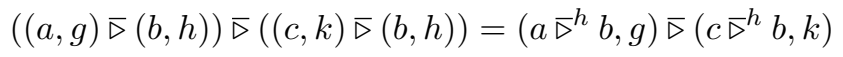

$$
\begin{aligned}
& =\left(\left(a \triangleright^{h} b\right) \triangleright^{k}\left(c \nabla^{h} b\right), g\right) \\
& =\left(\left(a \triangleright^{k} c\right) \triangleright^{k^{-1} h k}\left(b \unrhd^{k} c\right), g\right) \\
& =\left(a \bar{\triangleright}^{k} c, g\right) \bar{\triangleright}\left(b \unrhd^{k} c, k^{-1} h k\right) \\
& =((a, g) \bar{\triangleright}(c, k)) \bar{\triangleright}((b, h) \unrhd(c, k)) \text {. }
\end{aligned}
$$

Thus, $(Q, \unrhd, \bar{\nabla})$ is a biquandle.

Next, we note that

$$
(a, g) \cdot:\left(a \unrhd^{g} a, h\right) \mapsto(a, g)\left(a \unrhd^{g} a, h\right)=(a, g h)
$$

and

$$
\cdot\left(a \unrhd^{g} a, h\right):(a, g) \mapsto(a, g)\left(a \unrhd^{g} a, n\right)=(a, g h)
$$

are injective.

Since $a \unrhd^{g} a=b \unrhd^{g} a \Leftrightarrow a=b \Leftrightarrow b{\triangleright^{h}}^{h}=a{\triangleright^{h}}^{h}$, we have

$$
\begin{gathered}
((a, g),(b, h) \unrhd(a, g))=\left((a, g),\left(b \unrhd^{g} a, g^{-1} h g\right)\right) \in D \\
\Leftrightarrow((b, h),(a, g) \triangleright(b, h))=\left((b, h),\left(a \bar{\triangleright}^{h} b, g\right)\right) \in D .
\end{gathered}
$$

Then

$$
\begin{aligned}
(a, g)((a, h) \unrhd(a, g)) & =(a, g)\left(a \unrhd^{g} a, g^{-1} h g\right)=(a, h g) \\
& =(a, h)\left(a \triangleright^{h} a, g\right)=(a, h)((a, g) \triangleright(a, h)) .
\end{aligned}
$$


Since $((a, g) \unrhd(x, k),(b, h) \unrhd((x, k) \bar{D}(a, g)))=\left(\left(a \unrhd^{k} x, k^{-1} g k\right),\left(b \unrhd^{k}\left(x \bar{\triangleright}^{g} a\right), k^{-1} h k\right)\right)$, we have

$$
\begin{aligned}
((a, g) \unrhd(x, k),(b, h) \unrhd((x, k) \triangleright(a, g))) \in D & \Leftrightarrow\left(a \unrhd^{k} x\right) \unrhd^{k^{-1} g k}\left(a \unrhd^{k} x\right)=b \unrhd^{k}\left(x \bar{\triangleright}^{g} a\right) \\
& \Leftrightarrow\left(a \unrhd^{g} a\right) \unrhd^{k}\left(x \bar{\triangleright}^{g} a\right)=b \unrhd^{k}\left(x \bar{\triangleright}^{g} a\right) \\
& \Leftrightarrow a \unrhd^{g} a=b \\
& \Leftrightarrow((a, g),(b, h)) \in D .
\end{aligned}
$$

Then

$$
\begin{aligned}
(x, k) \unrhd\left((a, g)\left(a \unrhd^{g} a, h\right)\right) & =(x, k) \unrhd(a, g h) \\
& =\left(x \unrhd^{g h} a, h^{-1} g^{-1} k g h\right)=\left(\left(x \unrhd^{g} a\right) \unrhd^{h}\left(a \unrhd^{g} a\right), h^{-1} g^{-1} k g h\right) \\
& =\left(x \unrhd^{g} a, g^{-1} k g\right) \unrhd\left(a \unrhd^{g} a, h\right)=((x, k) \unrhd(a, g)) \unrhd\left(a \unrhd^{g} a, h\right)
\end{aligned}
$$

and

$$
\begin{aligned}
\left((a, g)\left(a \unrhd^{g} a, h\right)\right) \unrhd(x, k) & =(a, g h) \unrhd(x, k)=\left(a \unrhd^{k} x, k^{-1} g h k\right) \\
& =\left(a \unrhd^{k} x, k^{-1} g k\right)\left(\left(a \unrhd^{k} x\right) \unrhd^{k^{-1} g k}\left(a \unrhd^{k} x\right), k^{-1} h k\right) \\
& =\left(a \unrhd^{k} x, k^{-1} g k\right)\left(\left(a \unrhd^{g} a\right) \unrhd^{k}\left(x \bar{\triangleright}^{g} a\right), k^{-1} h k\right) \\
& =\left(a \unrhd^{k} x, k^{-1} g k\right)\left(\left(a \unrhd^{g} a, h\right) \unrhd\left(x \bar{\triangleright}^{g} a, k\right)\right) \\
& =((a, g) \unrhd(x, k))\left(\left(a \unrhd^{g} a, h\right) \unrhd((x, k) \triangleright(a, g))\right) .
\end{aligned}
$$

Since $((a, g) \bar{\triangleright}(x, k),(b, h) \bar{\triangleright}((x, k) \unrhd(a, g)))=\left(\left(a \bar{\triangleright}^{k} x, g\right),\left(b \bar{\triangleright}^{g^{-1} k g}\left(x \unrhd^{g} a\right), h\right)\right)$, we have

$$
\begin{aligned}
((a, g) \bar{\triangleright}(x, k),(b, h) \bar{\triangleright}((x, k) \unrhd(a, g))) \in D & \Leftrightarrow\left(a \bar{\triangleright}^{k} x\right) \unrhd^{g}\left(a \bar{\triangleright}^{k} x\right)=b \bar{\triangleright}^{g^{-1} k g}\left(x \unrhd^{g} a\right) \\
& \Leftrightarrow\left(a \unrhd^{g} a\right) \bar{\triangleright}^{g^{-1} k g}\left(x \unrhd^{g} a\right)=b \bar{\triangleright}^{g^{-1} k g}\left(x \unrhd^{g} a\right) \\
& \Leftrightarrow a \unrhd^{g} a=b \Leftrightarrow((a, g),(b, h)) \in D .
\end{aligned}
$$

Then

$$
\begin{aligned}
(x, k) \triangleright\left((a, g)\left(a \unrhd^{g} a, h\right)\right) & =(x, k) \bar{\triangleright}(a, g h) \\
& =\left(x \bar{\triangleright}^{g h} a, k\right)=\left(\left(x \bar{\triangleright}^{g} a\right) \bar{\triangleright}^{h}\left(a \unrhd^{g} a\right), k\right) \\
& =\left(x \bar{\triangleright}^{g} a, k\right) \bar{\triangleright}\left(a \unrhd^{g} a, h\right)=((x, k) \bar{\triangleright}(a, g)) \bar{\triangleright}\left(a \unrhd^{g} a, h\right),
\end{aligned}
$$

and

$$
\begin{aligned}
\left((a, g)\left(a \unrhd^{g} a, h\right)\right) \bar{\triangleright}(x, k) & =(a, g h) \bar{\triangleright}(x, k)=\left(a \bar{\triangleright}^{k} x, g h\right) \\
& =\left(a \bar{\triangleright}^{k} x, g\right)\left(\left(a \bar{\triangleright}^{k} x\right) \unrhd^{g}\left(a \bar{\triangleright}^{k} x\right), h\right) \\
& =\left(a \bar{\triangleright}^{k} x, g\right)\left(\left(a \unrhd^{g} a\right) \bar{\triangleright}^{g^{-1}} k g\left(x \unrhd^{g} a\right), h\right) \\
& =\left(a \bar{\triangleright}^{k} x, g\right)\left(\left(a \unrhd^{g} a, h\right) \bar{\triangleright}\left(x \unrhd^{g} a, g^{-1} k g\right)\right) \\
& =((a, g) \triangleright(x, k))\left(\left(a \unrhd^{g} a, h\right) \triangleright((x, k) \unrhd(a, g))\right) .
\end{aligned}
$$

Since $a \unrhd^{g h} a=\left(a \unrhd^{g} a\right) \unrhd^{h}\left(a \unrhd^{g} a\right)$, we have

$$
\begin{aligned}
((a, g),(b, h)),((a, g)(b, h),(c, i)) \in D & \Leftrightarrow a \unrhd^{g} a=b, a \unrhd^{g h} a=c \\
& \Leftrightarrow b \unrhd^{h} b=c, a \unrhd^{g} a=b \\
& \Leftrightarrow((b, h),(c, i)),((a, g),(b, h)(c, i)) \in D .
\end{aligned}
$$


Then

$$
((a, g)(b, h))(c, i)=(a, g h)(c, i)=(a, g h i)=(a, g)(b, h i)=(a, g)((b, h)(c, i)) .
$$

Finally, since $\left(a \unrhd^{g} a\right) \unrhd^{g^{-1} i}\left(a \unrhd^{g} a\right)=a \unrhd^{i} a$, there is an $(e, k) \in X \times G$ such that

$$
((a, g),(e, k)),((e, k),(d, j)) \in D, \quad(a, g)(e, k)=(c, i), \quad \text { and } \quad(e, k)(d, j)=(b, h)
$$

$\Leftrightarrow \exists e \in X, \exists k \in G$ such that $a \unrhd^{g} a=e, e \unrhd^{k} e=d, a=c, g k=i, e=b, k j=h$

$\Leftrightarrow\left(a \unrhd^{g} a\right) \unrhd^{g^{-1}} i\left(a \unrhd^{g} a\right)=d, a=c, g^{-1} i=h j^{-1}, a \unrhd^{g} a=b$

$\Leftrightarrow a \unrhd^{g} a=b, c \unrhd^{i} c=d, a=c, g h=i j$

$\Leftrightarrow((a, g),(b, h)),((c, i),(d, j)) \in D,(a, g)(b, h)=(c, i)(d, j)$

and $(Q, \unrhd, \bar{\nabla} \cdot \cdot)$ is a partially multiplicative biquandle.

Definition 5.5. For any $G$-family of biquandles $X$, the partially multiplicative biquandle $(Q, \unrhd, \bar{\triangleright}, \cdot)$ is the partially multiplicative biquandle associated to $X$.

Example 10. Let $\left(X,\left(\unrhd^{g}\right)_{g \in \mathbb{Z}_{4}},\left(\bar{\triangleright}^{g}\right)_{g \in \mathbb{Z}_{4}}\right)$ be the $\mathbb{Z}_{4}$-family of biquandles from Example 8, i.e. $X=\mathbb{Z}_{5}$ with $t=2$ and $s=3$. Then we have $Q=\mathbb{Z}_{5} \times \mathbb{Z}_{4}$ with operations

$$
\begin{aligned}
(a, g) \unrhd(b, h) & =\left(2^{h} a+\left(3^{h}-2^{h}\right) b, g\right) \\
(a, g) \bar{\triangleright}(b, h) & =\left(3^{h} a, g\right) \\
(a, g) \cdot\left(a \unrhd^{g} a, h\right) & =(a, g+h) .
\end{aligned}
$$

Then for instance, we have

$$
(2,3) \unrhd(3,3)=\left(2^{3}(2)+\left(3^{3}-2^{3}\right) 3,3\right)=(3,3) .
$$

\section{Counting Invariants}

Given a $Y$-oriented spatial trivalent graph diagram $\Gamma$ representing an $S^{1}$-oriented handlebody-knot and a partially multiplicative biquandle $X$, the number of $X$-colorings of semiarcs in a diagram of $\Gamma$ is unchanged by Reidemeister moves by construction. Thus we have

Definition 6.1. Let $\Gamma$ be a $Y$-oriented spatial graph diagram representing an $S^{1}$-oriented handlebody-knot and $X$ a partially multiplicative biquandle. Then the partially multiplicative biquandle counting invariant of $\Gamma$ with respect to $X$ is the number of $X$-colorings of $\Gamma$, denoted $\Phi_{X}^{\mathbb{Z}}(\Gamma)$.

Example 11. Let $X$ be the partially multiplicative biquandle associated to the Alexander biquandle $Z_{5}$ with $t=2$ and $s=3$ as in Example 8. Let us compute the number of $X$-colorings of the unknotted Theta graph $\Theta$ below:

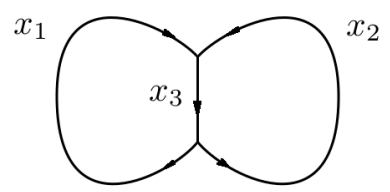

For any $x_{1}=(a, g) \in \mathbb{Z}_{5} \times \mathbb{Z}_{4}$, we must have $x_{2}=\left(a \unrhd^{g} a, h\right)$ and $x_{3}=(a, g h)$. Then for any choice of $g, h \in \mathbb{Z}_{4}$ and $a \in \mathbb{Z}_{5}$, we get a valid $X$-coloring; hence the counting invariant value is $\Phi_{X}^{\mathbb{Z}}(\Theta)=5(4)^{2}=80$. 
When $X$ is a $G$-family of biquandles, we can take advantage of this extra structure to enhance the counting invariant. Specifically, collecting together biquandle colorings which differ only in the the first component gives us a way of filtering the set of $X$-colorings of our handlebody-knot diagram which is unchanged by Reidemeister moves, since forgetting the first component yields a group coloring by $G$ (i.e., a group homomorphism from $\pi_{1}\left(S^{3} \backslash \Gamma\right)$ to $\left.G\right)$. Given a $G$-coloring $\psi \in \operatorname{Hom}\left(\pi_{1}\left(S^{3} \backslash \Gamma\right), G\right)$, let us denote by $\pi^{-1}(\psi)$ the set of $X$-colorings which project to $\psi$ by forgetting the first component $(a, g) \mapsto g$ on each semiarc. Then we have:

Definition 6.2. Let $X$ be a $G$-family of biquandles and $\Gamma$ a $Y$-oriented spatial trivalent graph diagram representing an $S^{1}$-oriented handlebody-knot. The $G$-enhanced biquandle counting invariant of $\Gamma$ is the polynomial

$$
\Phi_{X}^{G}(\Gamma)=\sum_{\psi \in \operatorname{Hom}\left(\pi_{1}\left(S^{3} \backslash \Gamma\right), G\right)} u^{\left|\pi^{-1}(\psi)\right|}
$$

Proposition 6.3. If two $Y$-oriented spatial trivalent graph diagrams $\Gamma$ and $\Gamma^{\prime}$ representing $S^{1}$-oriented handlebody-knots are related by Reidemeister moves, then for any $G$-family of biquandles $X$, we have

$$
\Phi_{X}^{G}(\Gamma)=\Phi_{X}^{G}\left(\Gamma^{\prime}\right) .
$$

Example 12. Let us illustrate the computation of the $G$-family enhanced counting invariant for the Kinoshita Theta graph $\Gamma$ below

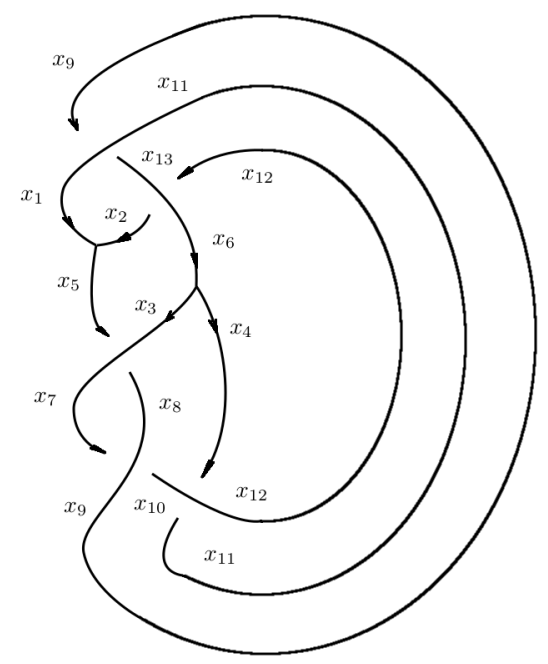

with respect to the $G$-family of biquandles associated to the Alexander biquandle $\mathbb{Z}_{3}$ with $t=1$ and $s=2$. We have

$$
\begin{array}{ll}
x \unrhd^{[1]} y=1 x+(2-1) y=x+y, & x \bar{\triangleright}^{[1]} y=2 x \\
x \unrhd^{[2]} y=1^{2} x+\left(2^{2}-1^{2}\right) y=x & x \bar{\triangleright}^{[2]} y=2^{2} x=x
\end{array}
$$

so $X$ has type 2 and we have a $\mathbb{Z}_{2}$-family of biquandles. Then we have partially multiplicative biquandle operations

$$
\begin{aligned}
(a, g) \unrhd(b, h) & =\left(a+\left(2^{h}-1\right) b, g\right) \\
(a, g) \bar{\triangleright}(b, h) & =\left(2^{h} a, g\right) \\
(a, g) \cdot\left(a \unrhd^{g} a, h\right) & =(a, g+h) .
\end{aligned}
$$

Since the group colorings don't change at crossings with this abelian group $G=\mathbb{Z}_{2}$, a choice of $g, h \in \mathbb{Z}_{2}$ 
determines the group coloring.

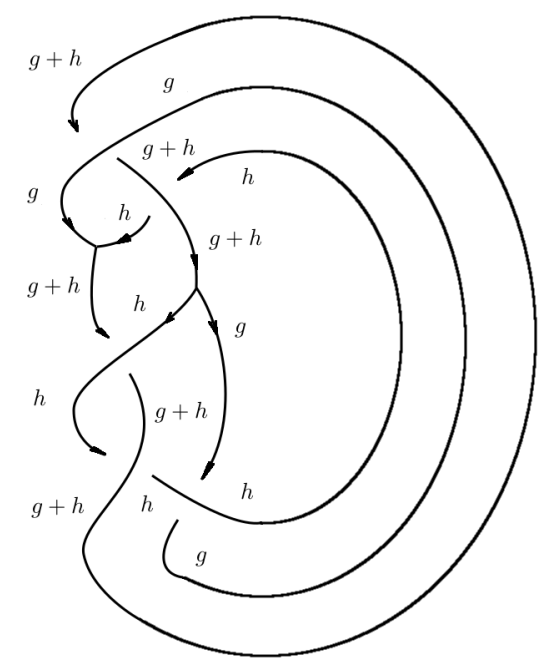

For each assignment of $g, h \in \mathbb{Z}_{2}$ to the second components of $x_{1}=\left(a_{1}, g\right)$ and $x_{2}=\left(a_{2}, h\right)$ respectively, we get a system of linear equations over $\mathbb{Z}_{3}$. For instance, taking $g=0$ and $h=1$, we have

$$
\begin{aligned}
a_{1} & =a_{5} \\
a_{2} & =a_{1} \unrhd^{g} a_{1} \\
a_{3} & =a_{6} \\
a_{3} & =a_{7} \bar{\triangleright}^{g+h} a_{5} \\
a_{4} & =a_{3} \unrhd^{h} a_{3} \\
a_{4} & =a_{11} \unrhd^{h} a_{10} \\
a_{6} & =a_{13} \bar{\triangleright}^{h} a_{2} \\
a_{8} & =a_{5} \unrhd^{h} a_{7} \\
a_{8} & =a_{9} \bar{\triangleright}^{h} a_{7} \\
a_{10} & =a_{7} \unrhd^{g+h} a_{9} \\
a_{11} & =a_{1} \bar{\triangleright}^{g+h} a_{9} \\
a_{12} & =a_{2} \unrhd^{g+h} a_{13} \\
a_{12} & =a_{10} \bar{\triangleright}^{g} a_{11} \\
a_{13} & =a_{9} \unrhd^{g} a_{1}
\end{aligned} \quad\left[\begin{array}{lllllllllllll}
2 & 0 & 0 & 0 & 1 & 0 & 0 & 0 & 0 & 0 & 0 & 0 & 0 \\
1 & 2 & 0 & 0 & 0 & 0 & 0 & 0 & 0 & 0 & 0 & 0 & 0 \\
0 & 0 & 2 & 0 & 0 & 1 & 0 & 0 & 0 & 0 & 0 & 0 & 0 \\
0 & 0 & 2 & 0 & 0 & 0 & 2 & 0 & 0 & 0 & 0 & 0 & 0 \\
0 & 0 & 2 & 2 & 0 & 0 & 0 & 0 & 0 & 0 & 0 & 0 & 0 \\
0 & 0 & 0 & 2 & 0 & 0 & 0 & 0 & 0 & 1 & 1 & 0 & 0 \\
0 & 0 & 0 & 0 & 0 & 2 & 0 & 0 & 0 & 0 & 0 & 0 & 2 \\
0 & 0 & 0 & 0 & 1 & 0 & 1 & 2 & 0 & 0 & 0 & 0 & 0 \\
0 & 0 & 0 & 0 & 0 & 0 & 0 & 2 & 2 & 0 & 0 & 0 & 0 \\
0 & 0 & 0 & 0 & 0 & 0 & 1 & 0 & 1 & 2 & 0 & 0 & 0 \\
2 & 0 & 0 & 0 & 0 & 0 & 0 & 0 & 0 & 0 & 2 & 0 & 0 \\
0 & 1 & 0 & 0 & 0 & 0 & 0 & 0 & 0 & 0 & 0 & 2 & 1 \\
0 & 0 & 0 & 0 & 0 & 0 & 0 & 0 & 0 & 1 & 0 & 2 & 0 \\
0 & 0 & 0 & 0 & 0 & 0 & 0 & 0 & 1 & 0 & 0 & 0 & 2
\end{array}\right]
$$

which row-reduces over $\mathbb{Z}_{3}$ to

$$
\left[\begin{array}{lllllllllllll}
1 & 2 & 0 & 0 & 0 & 0 & 0 & 0 & 0 & 0 & 0 & 0 & 0 \\
0 & 1 & 0 & 0 & 0 & 0 & 0 & 0 & 0 & 0 & 0 & 2 & 1 \\
0 & 0 & 1 & 0 & 0 & 2 & 0 & 0 & 0 & 0 & 0 & 0 & 0 \\
0 & 0 & 0 & 1 & 0 & 1 & 0 & 0 & 0 & 0 & 0 & 0 & 0 \\
0 & 0 & 0 & 0 & 1 & 0 & 0 & 0 & 0 & 0 & 0 & 2 & 1 \\
0 & 0 & 0 & 0 & 0 & 1 & 0 & 0 & 0 & 1 & 1 & 0 & 0 \\
0 & 0 & 0 & 0 & 0 & 0 & 1 & 0 & 1 & 2 & 0 & 0 & 0 \\
0 & 0 & 0 & 0 & 0 & 0 & 0 & 1 & 1 & 2 & 0 & 2 & 1 \\
0 & 0 & 0 & 0 & 0 & 0 & 0 & 0 & 1 & 0 & 0 & 0 & 0 \\
0 & 0 & 0 & 0 & 0 & 0 & 0 & 0 & 0 & 1 & 1 & 0 & 2 \\
0 & 0 & 0 & 0 & 0 & 0 & 0 & 0 & 0 & 0 & 1 & 1 & 2 \\
0 & 0 & 0 & 0 & 0 & 0 & 0 & 0 & 0 & 0 & 0 & 0 & 1 \\
0 & 0 & 0 & 0 & 0 & 0 & 0 & 0 & 0 & 0 & 0 & 0 & 0 \\
0 & 0 & 0 & 0 & 0 & 0 & 0 & 0 & 0 & 0 & 0 & 0 & 0
\end{array}\right]
$$


so we have $|\{0,1,2\}|=3 X$-colorings where $x_{1}=\left(a_{1}, 0\right)$ and $x_{2}=\left(a_{2}, 1\right)$. Repeating for $(g, h)=(1,1),(1,0)$ and $(0,0)$, we obtain $3 X$-colorings for each, so we have

$$
\Phi_{X}^{G}(\Gamma)=4 u^{3} .
$$

\section{Questions}

We end with some questions for future work.

What other enhancements of the $X$ counting invariant can be defined when $X$ is a partially multiplicative biquandle or a group decomposable biquandle? What about cocycle invariants in this setting?

\section{References}

[1] M. Elhamdadi and S. Nelson. Quandles - an introduction to the algebra of knots, volume 74 of Student Mathematical Library. American Mathematical Society, Providence, RI, 2015.

[2] R. Fenn and C. Rourke. Racks and links in codimension two. J. Knot Theory Ramifications, 1(4):343406, 1992.

[3] R. Fenn, C. Rourke, and B. Sanderson. Trunks and classifying spaces. Appl. Categ. Structures, 3(4):321$356,1995$.

[4] A. Ishii. The Markov theorems for spatial graphs and handlebody-knots with Y-orientations. Internat. J. Math., 26(14):1550116, 23, 2015.

[5] A. Ishii. A multiple conjugation quandle and handlebody-knots. Topology Appl., 196:492-500, 2015.

[6] A. Ishii, M. Iwakiri, Y. Jang, and K. Oshiro. A G-family of quandles and handlebody-knots. Illinois J. Math., 57(3):817-838, 2013.

[7] D. Joyce. A classifying invariant of knots, the knot quandle. J. Pure Appl. Algebra, 23(1):37-65, 1982.

[8] L. H. Kauffman and D. Radford. Bi-oriented quantum algebras, and a generalized Alexander polynomial for virtual links. In Diagrammatic morphisms and applications (San Francisco, CA, 2000), volume 318 of Contemp. Math., pages 113-140. Amer. Math. Soc., Providence, RI, 2003.

[9] V. Lebed. Qualgebras and knotted 3-valent graphs. Fund. Math., 230(2):167-204, 2015.

[10] S. V. Matveev. Distributive groupoids in knot theory. Mat. Sb. (N.S.), 119(161)(1):78-88, 160, 1982.

Institute of Mathematics

UNIVERSITY OF TSUKUBA

1-1-1 TENNODAI

TSUKUBA, IBARAKi 305-8571, JAPAN

Department of Mathematical Sciences

Claremont McKenna College

850 Columbia Ave.

Claremont, CA 91767, USA 